\title{
Silicon-on-insulator single channel-extraction filter for DWDM applications
}

\author{
C. Alonso-Ramos*, A. Ortega-Moñux*, I. Molina-Fernández*, A. Annoni, ${ }^{\dagger}$ A. Melloni ${ }^{\dagger}$, \\ M. Strain ${ }^{\ddagger}$, M. Sorel ${ }^{\S}$, P. Orlandi ${ }^{\uparrow}$, P. Bassi, ${ }^{\uparrow}$ F. Morichetti ${ }^{\dagger}$ \\ *Universidad de Málaga, ETSI Telecomunicación, Campus de Teatinos s/n, 29071 Málaga, España \\ Email: caar@ic.uma.es \\ ${ }^{\dagger}$ Dipartimento di Elettronica, Informazione e Bioingegneria - Politecnico di Milano, 20133 Milano, Italy \\ $\ddagger$ Institute of Photonics, The University of Strathclyde, Glasgow G4 0NW, United Kingdom \\ ${ }^{\S}$ School of Engineering, University of Glasgow, Glasgow G12 8LT, United Kingdom \\ `Dipartimento di Ingegneria dell'Energia Elettrica e dell'Informazione, Università di Bologna, Bologna 40136, Italy
}

\begin{abstract}
A novel silicon photonics filter enabling singlechannel-extraction over a wavelength range wider than the telecom C-band is proposed. An extraction bandwidth of only $15 \mathrm{GHz}$ is demonstrated with off-band rejection of more than 15 dB.
\end{abstract}

\section{INTRODUCTION}

Optical filters capable to extract a narrow-band signal from a grid of densely spaced channels are key components of wavelength division multiplexing systems (WDM). Add-drop architectures can be realized by embedding wavelength selective reflectors, such as Bragg gratings, inside a Mach-Zehnder interferometer (MZI) [1] or by using ring resonators arrangements [2]. To realize Single Channel-Extraction (SCE) filters, these schemes require either gratings with narrow reflection bandwidths, in the order of $25-50 \mathrm{GHz}$, or ring resonators with very large free spectral ranges (FSRs), extending across a wavelength range of several tens of $\mathrm{nm}$. These requirements are both difficult to fulfill, thereby making the implementation of single channel add-drop filters a challenging issue.

Recently, the integration of a Bragg reflector inside a ring resonator has been exploited to demonstrate narrow-band SCE with very large FSR [3], [4]. The Bragg-loaded ring resonator, whose structure is schematically depicted in Fig. 1(a), combines the periodic narrow-band response of the ring with the aperiodic wide-band Bragg reflector response, thus providing an aperiodic frequency-domain reflection with a single narrowband reflection peak. However, as in conventional ring resonator filters, the FSR of this device is limited by the minimum bending radius. Moreover, optical channels resonating in the ring at wavelengths outside the Bragg reflection band are affected by the cavity-enhanced propagation loss and by the dispersion of the ring resonator.

In this work, we propose a novel SCE filter architecture that enables single channel-extraction over a wavelength range wider than the telecom C-band. Proof-of-concept devices, realized on a conventional SOI platform, demonstrate the possibility of extracting channels with bandwidths as narrow as $15 \mathrm{GHz}$ with off-band rejection better than $15 \mathrm{~dB}$.

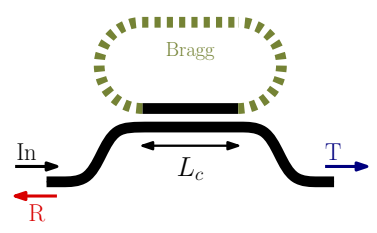

a)

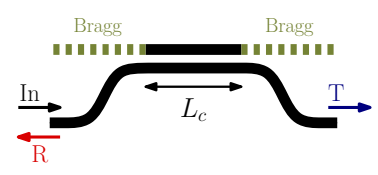

b)
Fig. 1. Schematic representation of: (a) Bragg-loaded ring resonator, (b) proposed Bragg-loaded directional coupler.

\section{SCE CONCEPT AND DESIGN}

Figure 1(b) shows the schematic of the proposed SCE filter structure. The filter is composed by a directional coupler (DC) with the two upper arms loaded with identical Bragg reflectors. At wavelengths within the grating reflection bandwidth, partial reflections in each loaded arm of the DC interfere producing a cavity-like behavior. Compared to the device of Fig. 1(a), the ring resonator is replaced with a Fabry-Pérot resonator, thus removing the FSR constraints due to the bending radius. The cavity length is determined by the DC length $L_{c}$ and by the penetration length of the field in the Bragg gratings [5]. In order to achieve SCE response, $L_{c}$ has to be designed in order to align the cavity resonance with the center of the Bragg reflection band. Outside the reflection band, no power is reflected back from the upper DC arms, so that the cavity effect disappears. In this way, off-band channels do not suffer from cavity-enhanced loss and dispersion, as it happens in the Bragg-loaded ring of Fig. 1(a). To avoid spurious resonance effects outside the grating bandwidth, impedance matching has to be achieved at the end of the DC arms; this can be done, for example, by narrowing the waveguides in order to make the light leak into the chip substrate.

Figure 2 shows the simulated response of a SCE filter (red curve) with DC coupling ratio $K=0.1$ that integrates Bragg reflectors (green curve) with index modulation $\delta n=0.01$ and 380 periods. The grating bandwidth $(6 \mathrm{~nm})$, evaluated as the distance between the first reflection zeros, is smaller than twice the FSR of the cavity $(8 \mathrm{~nm})$, thereby providing SCE operation. The resulting SCE reflection bandwidth, that 


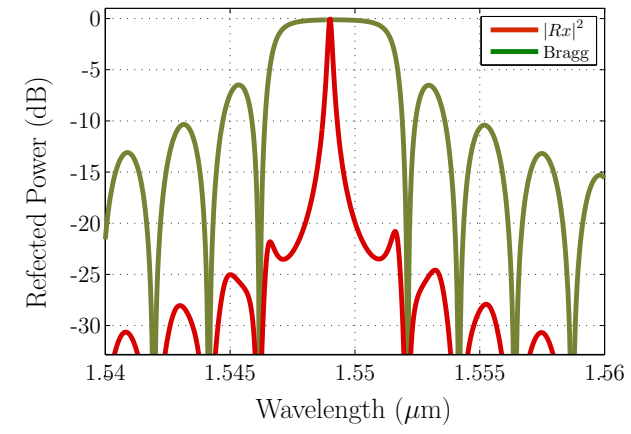

Fig. 2. Simulated reflection $|R x|^{2}$ (red curve) of the proposed SCE with DC coupling ratio $K=0.1$. The reflection spectrum of the Bragg gratings, with index modulation $\delta n=0.01$ and 380 periods, is shown by the green curve.

in the considered example is $27 \mathrm{GHz}$ wide, is determined by the cavity finesse, and thus by $K$ and the cavity loss.

\section{EXPERIMENTAL RESUlts}

The feasibility of the proposed SCE filter was demonstrated on a standard $220 \mathrm{~nm}$ SOI platform. Silicon waveguides with $220 \mathrm{~nm}$ thickness and $480 \mathrm{~nm}$ width, and covered with a 1 $\mu \mathrm{m}$ thick silica cladding, were fabricated by electron-beam lithography [6]. In the realized device, the length and the gap of the DCs are $L_{c}=10 \mu \mathrm{m}$ and $g=300 \mathrm{~nm}$. The Bragg gratings have a sinusoidal profile with a $5 \mathrm{~nm}$ peakto-peak depth $(475<w<485)$, a period $\Lambda=320 \mathrm{~nm}$ and 380 periods. In order to maximize the FSR of the cavity, the gratings are directly connected to the straight coupling region of the DC; on the other waveguide of the DC, transition regions with $20 \mu \mathrm{m}$ bending radius were realized. The device footprint, excluding the tapered waveguides cascaded to the gratings, is $250 \mu \mathrm{m} \times 20 \mu \mathrm{m}$. Tapered lensed fibers are used to inject and to collect light from the chip. Light generated by a tunable laser goes through a polarization-controller that sets a transverse electric (TE) polarization at the chip input with more than $30 \mathrm{~dB}$ extinction ratio. An optical circulator is used to extract the reflection spectrum $\left|R_{x}\right|^{2}$ of the filter. The measured spectrum is processed using coherent optical frequency-domain reflectometry [7] to remove the Fabry-Pérot ripples produced by facet reflections.

Fig. 3(a) shows the measured spectral response in 1530$1570 \mathrm{~nm}$ wavelength range. Only a single reflection peak is observed at $1550.1 \mathrm{~nm}$, with no evidence of side reflection peaks. Filter isolation is limited to about $20 \mathrm{~dB}$ due to the offband roughness-induced backscattering of the bus waveguide [7]. The reflection spectrum $\left|R_{x}\right|^{2}$ [see Fig. 3(b)] has a remarkable narrow bandwidth of $B W_{3 \mathrm{~dB}} \sim 15 \mathrm{GHz}$ with a rejection of about $15 \mathrm{~dB}$. Further, in the transmission spectrum $\left|T_{x}\right|^{2}$ no off-band notches are observed, demonstrating that outside the grating band no significant cavity effects occurs. These results demonstrate the feasibility of the proposed scheme.

\section{CONCLUSION}

A novel resonator architecture has been proposed for the implementation of SCE filters for WDM and DWDM systems.
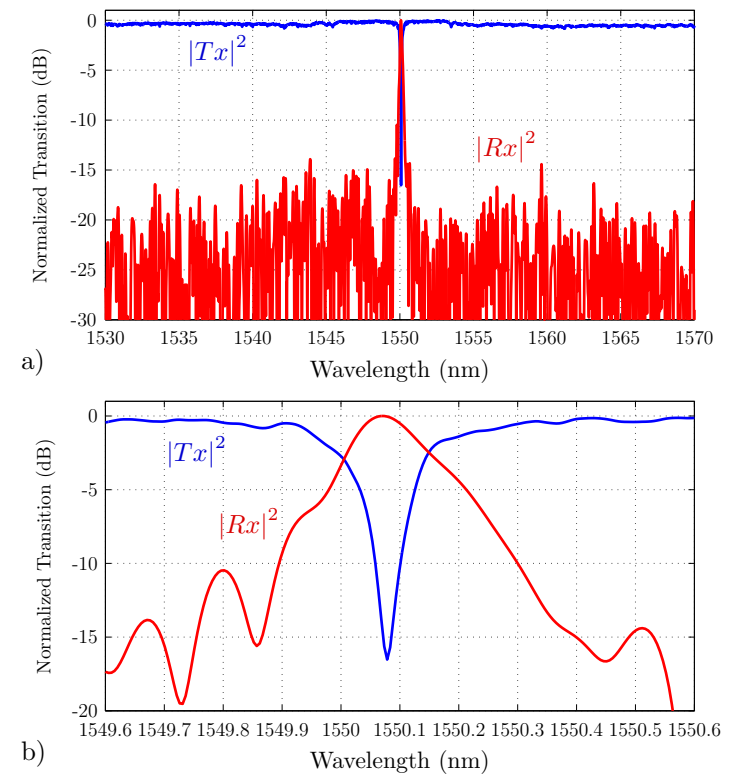

Fig. 3. Normalized measured transmission $\left|T_{x}\right|^{2}$ (blue curve) and reflection $\left|R_{x}\right|^{2}$ (red curve) of fabricated SCE filter: spectral response over (a) the full C-band, and (b) a wavelength range of $1 \mathrm{~nm}$ centered around $1550.1 \mathrm{~nm}$.

The Bragg-loaded DC structure obviates the minimum bending radii limitations of SCE schemes based on ring resonators and avoids unwanted cavity-enhanced loss distortion in off-band WDM channels. A single reflected band of $15 \mathrm{GHz}$ bandwidth with a $15 \mathrm{~dB}$ rejection has been demonstrated. The proposed SCE filter can be inserted in the arms of a MZI to remove the need for a circulator.

\section{ACKNOWLEDGMENTS}

This work was partially supported by the Italian PRIN 2009 project SAPPHIRE and by the FP7-ICT European FET Project BBOI. The Authors gratefully acknowledge the James Watt Nanofabrication Centre (JWNC) staff at the University of Glasgow for the fabrication of the devices.

\section{REFERENCES}

[1] H. Yamada et al. , "Optical add-drop multiplexers based on Si-wire waveguides," Appl. Phys. Lett., vol. 86, no. 19, pp. 19110-1-19110-3, May 2005.

[2] A. Melloni et al. , "Synthesis of Direct-Coupled-Resonators Bandpass Filters for WDM Systems," J. Lightwave Technol., vol. 20, no. 2, pp. 296-303, Feb. 2002.

[3] A. Arbabi et al. , "Realization of a narrowband single wavelength microring mirror," Appl. Phys. Lett., vol. 99, no. 9, pp. 091105-1-0911053, Aug. 2011.

[4] Y. Mo Kang et al., "Engineering the spectral reflectance of microring resonators with integrated reflective elements," Opt. Express, vol. 18, no. 16, pp. 16813-16825, Aug. 2010.

[5] A. Melloni et al., "Equivalent circuit of Bragg gratings and its application to FabryPérot cavities," J. Opt. Soc. Am. A, vol. 20, no. 2, pp. 273-281, Feb. 2003.

[6] M. Gnan et al., "Fabrication of low-loss photonic wires in silicon-oninsulator using hydrogen silsesquioxane electron-beam resist," Electr. Lett., vol. 44, no. 2, pp. 115-116, Jan. 2008.

[7] F. Morichetti et al., "Roughness induced backscattering in optical silicon waveguides," Phys. Rev. Lett., vol. 104, pp. 033902-1-033902-4, Jan. 2010 . 